\title{
STABILITY AND DIMENSION-A COUNTEREXAMPLE TO A CONJECTURE OF CHOGOSHVILI
}

\author{
YAKI STERNFELD
}

\begin{abstract}
For every $n \geq 2$ we construct an $n$-dimensional compact subset $X$ of some Euclidean space $E$ so that none of the canonical projections of $E$ on its two-dimensional coordinate subspaces has a stable value when restricted to $X$. This refutes a longstanding claim due to Chogoshvili. To obtain this we study the lattice of upper semicontinuous decompositions of $X$ and in particular its sublattice that consists of monotone decompositions when $X$ is hereditarily indecomposable.
\end{abstract}

\section{INTRODUCTION}

Let $X$ and $Y$ be subsets of some Euclidean space $E . \quad X$ is said to be removable from $Y$ if for every $\varepsilon>0$ there is a map $f: X \rightarrow E$ with $\| x-$ $f(x) \|<\varepsilon$ so that $f(X) \cap Y=\varnothing$.

In [Ch] Chogoshvili claims that if $X \subset E_{m}$ ( $=m$-dimensional Euclidean space) is $n$-dimensional then there exists an $(m-n)$-dimensional affine subspace $A$ of $E_{m}$ so that $X$ is not removable from $A$. Moreover, given a coordinate system in $E_{m}, A$ can be chosen to be parallel to one of the $(m-n)$-dimensional linear subspaces determined by this coordinate system (Remark III, p. 292 in [Ch]).

Recently R. Pol discovered a gap in Chogoshvili's proof which went unnoticed for many years (see $\left.\left[E_{1}\right]\right)$. Sitnikov's example [Si] demonstrates that the theorem fails if $X$ is not assumed to be compact. The main goal of this article is to present a compact counterexample to the stronger version of Chogoshvili's theorem. We shall prove:

Theorem 1.1. For every $n \geq 2$ there exists an $n$-dimensional compact subset $X$ of some m-dimensional Euclidean space $E$ ( $m$ depends on $n)$, and a representation $E=\sum_{a} \oplus E_{a}$ of $E$ as a direct sum of (mutually orthogonal) linear subspaces $E_{a}$ of the same dimension $l$ each, so that the following holds: for every choice of a basis $\left\{e_{a, i}\right\}_{i=1}^{l}$ for $E_{a}, X$ is removable from every $(m-2)$-dimensional affine subspace of $E$ which is parallel to one of the $(m-2)$-dimensional linear subspaces determined by the basis $\bigcup_{a}\left\{e_{a, i}\right\}_{i=1}^{l}$ of E.

Remark. By a basis here we mean of course a linear basis and $L$ is an $(m-2)$ dimensional linear subspace determined by the basis if it is the linear span of $m-2$ (i.e. all but 2) of its elements.

Received by the editors September 1, 1991.

1991 Mathematics Subject Classification. Primary 54F45. 
This stronger than just a counterexample to the Chogoshvili claim: first, the affine subspaces from which $X$ is removable are not merely $(m-n)$-dimensional but $(m-2)$-dimensional (even for $n>2)$ and also, as $l>1$, there is a degree of freedom in the choice of the bases $\left\{e_{a, i}\right\}_{i=2}^{l}$ for $E_{a}$. Note however that the decomposition $E=\sum_{a} \oplus E_{a}$ is fixed, so that we do not have a counterexample for the weaker version of the Chogoshvili theorem which remains unsettled. The example, to be constructed in $\S 5$, will actually possess even stronger properties which for the sake of convenience will be stated there. In $\S 2$ we introduce the concept of $n$-stable mappings and study their relevant properties. In $\S 3$ we present the lattice $\operatorname{DEC}(X)$ of upper semicontinuous decompositions of a compact metrizable space $X$, and in $\S 4$ we study its sublattice $M(X)$ which consists of the monotone decompositions when $X$ is a hereditarily indecomposable continuum.

We give detailed proofs only to results which are applied in the construction. We state without proofs some other results which arise naturally. These are marked by $\mathrm{a}^{*}$ and will be proved elsewhere.

The space $X$ in the example is an $n$-dimensional hereditarily indecomposable (H.I.) continuum. Such spaces were first constructed by Bing [B], and were recently applied by $\mathrm{R}$. Pol [P] to construct subsets of Cartesian product spaces with some remarkable properties.

Finally I wish to thank Mr. M. Levin for valuable conversations.

\section{2. n-STABLE MAPPINGS}

Let $f: X \rightarrow Y$ be a mapping. (All spaces in this article are assumed to be metrizable, and all maps are assumed to be continuous.) Recall that a point $y \in Y$ is called a stable value of $f$ if there exists $\varepsilon>0$ such that for all $g: X \rightarrow Y$ with $d(f(x), g(x))<\varepsilon$ for all $x$ in $X, y \in g(X)$.

Let $B_{n}$ be a closed $n$-ball in $n$-dimensional Euclidean space $E_{n}$ with boundary $S_{n-1}$. A mapping $f: X \rightarrow B_{n}$ is essential if the map $f / f^{-1}\left(S_{n-1}\right)$ : $f^{-1}\left(S_{n-1}\right) \rightarrow S_{n-1}$ is not extendable to a map $g: X \rightarrow S_{n-1}$. It is well known that a map $f: X \rightarrow E_{n}$ has a stable value if and only if it maps essentially onto some $n$-ball $B_{n} \subset E_{n}$ (i.e. $f / f^{-1}\left(B_{n}\right): f^{-1}\left(B_{n}\right) \rightarrow B_{n}$ is essential).

It is easy to see that the Chogoshvili claim is equivalent to the following: "Let $X \subset E_{m}$ be $n$-dimensional. Then there exists an $n$-dimensional linear subspace $L_{n}$ of $E_{m}$ such that the restriction to $X$ of the orthogonal projection $P: E_{m} \rightarrow L_{n}$ has a stable value." And the stronger version is as above with $L_{n}$ a subspace determined by the coordinate system and $P$ a coordinate projection.

The following is a more general concept of stability which turns out to be useful.

Definition. A map $f: X \rightarrow Y$ is said to be $n$-stable if for every metric space $W$ which contains $Y$ there exists a neighbourhood $U$ of $f$ in $C(X, W)$ so that $\operatorname{dim} g(X) \geq n$ for all $g$ in $U$. If $f$ is not $n$-stable then we call it $n$-unstable.

We apply this definition for compact spaces $X$, in which case the topology on $C(X, W)$ is induced by the metric $\zeta(f, g)=\sup \{d(f(x), g(x)): x \in X\}$ where $d$ is a metric on $W$. Through the rest of this section we assume that $X$ is compact. 
Proposition 2.1. A map $f: X \rightarrow E_{n}$ is $n$-stable if and only if it has a stable value.

Proof. 1. Let $f: X \rightarrow E_{n}$ have a stable value. Without loss of generality we may assume that $f$ maps essentially onto some $n$-ball $B_{n}$. Let $W$ be a metric space that contains $F(X)=B_{n}$. Again we may assume that $W$ is a Banach space. There exists a retraction $r: W \rightarrow B_{n}$ so that $\|x-r(x)\| \leq$ $2 d\left(x, B_{n}\right), x \in W$. (See [B.P, p. 61].) Let $\varepsilon$ be positive. If there exists some $g_{\varepsilon}: X \rightarrow W$ with $\left\|f-g_{\varepsilon}\right\|<\varepsilon$ and $\operatorname{dim} g_{\varepsilon}(X) \leq n-1$ then $r: g_{\varepsilon}(X) \rightarrow$ $B_{n}$ can be approximated by some $r_{\varepsilon}: g_{\varepsilon}(X) \rightarrow B_{n}$ with $\left\|r-r_{\varepsilon}\right\|<\varepsilon$ and $\operatorname{dim} r_{\varepsilon} g_{\varepsilon}(X) \leq n-1$. It follows that $f_{\varepsilon}=r_{\varepsilon} g_{\varepsilon}: X \rightarrow B_{n}$ satisfies $\left\|f-f_{\varepsilon}\right\|<5 \varepsilon$ and $\operatorname{dim} f_{\varepsilon}(X) \leq n-1$. For sufficiently small $\varepsilon$ however this is impossible since as $f$ maps essentially on $B_{n}, f_{\varepsilon}(X)$ must contain some $n$-ball, and it follows that $f$ is $n$-stable.

2. Let $f: X \rightarrow E_{n}$ be $n$-stable, and let us assume that $f(X)$ is contained in some $n$-ball $B_{n}$. If $f$ has no stable values then by a standard argument for every finite subset $F$ of $E_{n}$ and every $\varepsilon>0$ there exists a map $g=$ $g_{F, \varepsilon}: X \rightarrow B_{n}$ with $\|f-g\|<\varepsilon$ and $g(X) \subset B_{n} \backslash F$. As for every $\varepsilon>0$ there exist a finite subset $F_{\varepsilon}$ of $B_{n}$ and a retraction $r_{\varepsilon}: B_{n} \backslash F_{\varepsilon} \rightarrow Y$, with $\operatorname{dim} Y \leq n-1$ and $\left\|x-r_{\varepsilon} x\right\|<\varepsilon, f_{\varepsilon}=r_{\varepsilon} g$ approaches $f$ as close as we please, and $\operatorname{dim} f_{\varepsilon}(X) \leq n-1$. Hence $f$ must be $n$-unstable.

Proposition 2.2*. A map $f: X \rightarrow Y$ is n-stable if and only if for every $W \supset Y$ there exists a neighborhood $U$ of $f$ in $C(X, W)$ so that $\inf \left\{d_{n} g(X): g \in\right.$ $U\}>0 .\left(d_{n}(\cdot)\right.$ is the n-dimensional degree as defined in $\left.[\mathrm{Ku}, p .105].\right)$

Proposition 2.3. Let $f: X \rightarrow Y$ be k-unstable. Then for every $g: Y \rightarrow Z$ $h=g f: X \rightarrow Z$ is $k$-unstable.

Proof. We may assume that $Y$ is compact. Let $\varepsilon$ be positive. Let $Y_{1}$ be a Banach space which contains $Y$. Let $Y_{2}$ denote the closed convex hull of $Y$ in $Y_{1} . \quad Y_{2}$ is compact. Let $Z_{1}$ be a Banach space that contains $Z$ and let $\hat{g}: Y_{2} \rightarrow Z_{1}$ extend $g$. Let $\delta>0$ be so small that $\left\|y_{1}-y_{2}\right\|<3 \delta$ in $Y_{2}$ implies that $\left\|\hat{g}\left(y_{1}\right)-\hat{g}\left(y_{2}\right)\right\|<\varepsilon$ in $Z_{1}$. As $f$ is $k$-unstable there exists some Banach space $Y_{3}$ containing $Y_{1}$ and a map $f^{\prime}: X \rightarrow Y_{3}$ with $\left\|f-f^{\prime}\right\|<\delta$ and $\operatorname{dim} f^{\prime}(X) \leq k-1$. By a standard argument there exists a $2 \delta$-translation $f^{\prime \prime}$ of $f^{\prime}(X)$ into some $(k-1)$-dimensional polyhedron $H$ contained in $Y_{2}$. (Just pick the vertices of $H$ in $Y$.) Then $\left\|f-f^{\prime \prime} f^{\prime}\right\|<3 \delta$ and by the choice of $\delta\left\|g f-\hat{g} f^{\prime \prime} f^{\prime}\right\|=\left\|\hat{g} f-\hat{g} f^{\prime \prime} f^{\prime}\right\|<\varepsilon$. As $H=f^{\prime \prime} f^{\prime}(X)$ is $(k-1)$-dimensional there exists some map $l: H \rightarrow Z_{1}$ with $\operatorname{dim} l(H) \leq k-1$ and $\|l-\hat{g}\|<\varepsilon$ on $H$. Then $\operatorname{dim} l f^{\prime \prime} f^{\prime}(X) \leq k-1$ and $\left\|h-l f^{\prime \prime} f^{\prime}\right\| \leq\left\|h-\hat{g} f^{\prime \prime} f^{\prime}\right\|+\left\|\hat{g} f^{\prime \prime} f^{\prime}-l f^{\prime \prime} f^{\prime}\right\|<$ $\varepsilon+\varepsilon=2 \varepsilon$, so $h$ is $k$-unstable.

Proposition 2.3 implies in particular that a map which factors through some $(k-1)$-dimensional spaces is $k$-unstable. It is thus natural to ask whether every $k$-unstable map must factor through some $(k-1)$-dimensional space. The answer is negative for $k \geq 2$ and affirmative for $k=1$. (The domain is assumed to be compact.)

Proposition 2.4*. A 1-unstable map factors through some 0-dimensional space. For $k \geq 2$ there exist $k$-unstable maps which do not factor through any $(k-1)$ dimensional space. 
Proposition 2.5*. A light map on a $k$-dimensional space is $k$-stable.

Actually much stronger results hold:

Theorem 2.1*. Let $f: X \rightarrow Y$ be a map and let $Y_{k}=\left\{y \in Y: \operatorname{dim} f^{-1}(y) \geq\right.$ $k\}$. If $\operatorname{dim} X>\max \left\{k+\min \left\{n-1, \operatorname{dim} Y_{k}\right\}, k=0,1, \ldots, \operatorname{dim} f\right\}$ then $f$ is $n$-stable. (This is a "stable" version of Vainstein's theorem $\left[\mathrm{E}_{2}, p .283\right]$.) Theorem 2.1 implies in particular

Proposition 2.6*. Let $f: X \rightarrow Y$ be a map. If

$$
\operatorname{dim} X>\operatorname{dim} f+\operatorname{dim}\left\{y \in Y: \operatorname{dim} f^{-1}(y)>\operatorname{dim} X-n\right\}
$$

then $f$ is $n$-stable.

This can be applied to prove the following Chogoshvili-type theorem.

Theorem 2.2*. Let $X$ be an $n$-dimensional compact subset of $E_{m}$. Then one of the $(m-1)$-dimensional coordinate projections is $n$-stable on $X$.

\section{THE LATTICE OF UPPER SEMICONTINUOUS DECOMPOSITIONS}

In this section we assume that $X$ is a compact space, and that the range of the maps we consider on $X$ is metrizable. We let $f: X \rightarrow Y$ be a map. Then $f$ induces an upper semicontinuous (u.s.c.) decomposition $\left\{f^{-1}(y): y \in\right.$ $f(X)\}$ on $X$ (see [Ku, p. 65]) and conversely, the quotient map of each u.s.c. decomposition of $X$ has a compact metrizable range.

Let $f$ and $g$ be two maps on $X$. We let $f \sim g$ if $f$ and $g$ induce the same u.s.c. decomposition on $X$. Evidently this occurs if and only if there exists a homeomorphism $h: f(X) \rightarrow g(X)$ such that the diagram

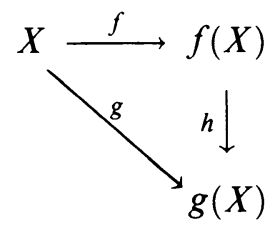

commutes.

Let $\operatorname{DEC}(X)$ denote the set of all maps on $X$ modulo the equivalence relation $\sim$. Clearly the elements of $\operatorname{DEC}(X)$ can be regarded as u.s.c. decompositions of $X$, but we shall still use functional notation. For $f, g$ in $\operatorname{DEC}(X)$ set $f \leq g$ if the decomposition of $X$ induced by $f$ refines that of $g$. This is equivalent to the existence of an $h$ which is not necessarily a homeomorphism in the above diagram. In that case we say that $f$ refines $g$ or that $g$ is coarser that $f$.

It follows from Proposition 2.3 that if $f \leq g$ and if $f$ is $k$-unstable then so is $g$. In particular, $f: X \rightarrow Y$ is $k$-unstable if and only if all the maps in the equivalence class mod $\sim$ of $f$ are $k$-unstable. For $f, g$ in $\operatorname{DEC}(X)$ let $f \wedge g=h$ be the decomposition of $X$ which, at $x \in X$ is the intersection of the fibers of $f$ and $g$ at $x$. Thus $h^{-1} h(x)=f^{-1} f(x) \cap g^{-1} g(x)$. As a map $h$ may be regarded as $h: X \rightarrow f(X) \times g(X)$ with $h(x)=(f(x), g(x))$. Similarly we define the inf of any set $\left\{f_{a}\right\}_{a \in A} \subset \operatorname{DEC}(X)$ by $f=\Lambda_{a} f_{a}$, $f^{-1} f(x)=\bigcap_{a} f_{a}^{-1} f_{a}(x)$, and $f$ can be regarded as a map from $X$ into $\pi_{a} f_{a}(X)$ whose $a$ coordinate is $f_{a}$. Clearly $\bigwedge_{a} f_{a} \leq f_{a}$ for all $a$ and if $g \in \operatorname{DEC}(X)$ 
satisfies $g \leq f_{a}$ for all $a$ then $g \leq \bigwedge_{a} f_{a}$. So $\Lambda$ is actually an inf. We may define $\bigvee F$ for $F \subset \operatorname{DEC}(X)$ as $\bigvee F=\bigwedge\{g: f \leq g$ for all $f$ in $F\}$. $\operatorname{DEC}(X)$ with these operations is a complete lattice. Note however that unlike the $\Lambda$ operation which has a simple explicit representation in terms of intersections, the structure of $f \vee g$ is not clear at all. In the next section we shall see that under certain restrictions $f \vee g$ also has a simple structure.

Fix a positive integer $k$. For $f \in \operatorname{DEC}(X)$ define

Definition 3.1. $f^{*}=\{g: g \in \operatorname{DEC}(X), g \leq f, g$ is $k$-unstable $\}$. By Proposition $2.3 f^{*}$ is empty if and only if $f$ is $k$-stable.

Proposition 3.1. Let $f, g \in \operatorname{DEC}(X) . f \wedge g$ is $k$-stable if and only if $f^{*} \cap g^{*}=$ $\varnothing$.

Proof. If $f \wedge g$ is $k$-stable then $f^{*} \cap g^{*}=\varnothing$ since if $h \in f^{*} \cap g^{*}$ then $h \leq f \wedge g$ is $k$-unstable and $f \wedge g$ must be $k$-unstable too. If $f^{*} \cap g^{*}=\varnothing$ then $f \wedge g$ must be $k$-stable since otherwise $f \wedge g$ would be in $f^{*} \cap g^{*}$.

The results in the rest of this section are not applied in the construction.

Proposition 3.2*. If $f$ is $k$-unstable then $f^{*}$ contains minimal elements, i.e. elements $g$ so that $h \leq g, h \neq g$ implies that $h$ is $k$-stable.

Let $U$ be a (finite open) cover of $X$. For $f \in \operatorname{DEC}(X)$ set $f \leq U$ if $f$ refines $U$.

Theorem 3.1*. $f$ is $k$-unstable if and only if every cover $U$ such that $f \leq U$ has a refinement $V$ of order $\leq k$.

Remark. Note that it is not required that $f \leq V$. (This would imply that $\operatorname{dim} f(X)<k$.)

Proposition 3.3*. For $k>1$ there exist minimal $k$-unstable elements of $f$ of $\operatorname{DEC}(X)$ with $\operatorname{dim} f(X)=k$. (Compare with Proposition 2.4*.)

The families $U=\{f \in \operatorname{DEC}(X): f \leq U\}, U$ a cover of $X$, form a basis for a topology on $\operatorname{DEC}(X)$. This topology is rather trivial. (Note that the class of the embeddings is an element of very open set.) However

Theorem 3.2*. On the set of minimal k-unstable elements of $\operatorname{DEC}(X)$ this topology is a Hausdorff topology. (Note that if $\operatorname{dim} X<k$ then there is only one minimal $k$-unstable element namely the class of the embeddings.)

\section{THE LATTICE OF MONOTONE U.S.C. DECOMPOSITIONS OF A HEREDITARILY INDECOMPOSABLE CONTINUUM}

In this section $X$ is assumed to be an H.I. continuum.

Thus if $F$ and $H$ are subcontinua of $X$ so that $H \cap F \neq \varnothing$ then either $H \subset$ $F$ or $F \subset H$. (Since otherwise $H \cup F$ would be a decomposable continuum.) It follows that

Proposition 4.1. Every family of subcontinua of $X$ with a nonempty intersection is totally ordered by inclusion.

An element $f$ of $\operatorname{DEC}(X)$ is monotone if $f^{-1}(y)$ is a continuum for all $y$ in $f(X)$. Let $M(X)$ denote the set of all monotone elements of $\operatorname{DEC}(X)$. In general $M(X)$ is not a sublattice of $\operatorname{DEC}(X)$ but when $X$ is H.I. then it is. 
Proposition 4.2. Let $\left\{f_{a}\right\}_{a \in A} \subset M(X)$. Then $f=\bigwedge_{a} f_{a} \in M(X)$.

Proof. $f^{-1} f(x)=\bigcap_{a} f_{a}^{-1} f_{a}(x)$. All the continua $f_{a}^{-1} f_{a}(x)$ contain $x$. Thus by Proposition 4.1 they are totally ordered by inclusion and hence their interaction is a continuum.

Definition 4.1. For $f, g$ in $M(X)$ let $h=f \vee g$ be defined by $h^{-1} h(x)=$ $f^{-1} f(x) \cup g^{-1} g(x)$.

Proposition 4.3. $h=f \vee g$ is a well-defined element of $M(X)$ and it agrees with the earlier definition of $f \vee g$ in $\operatorname{DEC}(X)$ namely $\wedge\{l: f \leq l, g \leq l\}$.

Proof. By Proposition 4.1 $h^{-1} h(x)$ is the larger among $f^{-1} f(x)$ and $g^{-1} g(x)$. It follows that 4.1 actually defines a closed decomposition of $X$. We check that it is u.s.c. Let $\left\{u_{n}\right\}_{n=1}^{\infty}$ be a sequence of fibers of $h$ which converges to some elements $u$ in $2^{X}$. We must show that $u$ is contained in some fiber of $h$.

Each $u_{n}$ is either a fiber of $f$ or a fiber of $g$ and as $f$ and $g$ are u.s.c. $u$ must be contained in a fiber of $f$ or in a fiber of $g$. So, if $x \in u$ then $u \subset f^{-1} f(x)$ or $u \subset f^{-1} g(x)$ and thus $u \subset f^{-1} f(x) \cup g^{-1} g(x)=h^{-1} h(x)$, and it follows that $h$ is u.s.c. Thus $h \in M(X)$. If $f \leq l$ and $g \leq l$ then $f \vee g \leq l$, also $f \leq f \vee g$ and $g \leq f \vee g$. It follows that $h=\bigwedge\{l: l \in \operatorname{DEC}(X)$, $f \leq l, g \leq l\}$.

Definition 4.2. For $f \in \operatorname{DEC}(X)$ let the singular set $S_{f}$ of $f$ be defined by $S_{f}=\left\{x \in X: f^{-1} f(x)=\{x\}\right\}$.

Proposition 4.4. Let $f, g \in M(X)$. Then $S_{f \wedge g}=S_{f} \cup S_{g}$ and $S_{f \vee g}=S_{f} \cap S_{g}$. Proof. Let $h=f \wedge g . h^{-1} h(x)=f^{-1} f(x) \cap g^{-1} g(x)$ so clearly $h^{-1} h(x)=\{x\}$ if and only if either $f^{-1} f(x)=\{x\}$ or $g^{-1} g(x)=\{x\}$ (recall that one of $f^{-1} f(x)$ and $g^{-1} g(x)$ contains the other) and it follows that $S_{h}=S_{f} \cup S_{g}$.

Let $l=f \vee g . l^{-1} l(x)=f^{-1} f(x) \cup g^{-1} g(x)$ and thus $l^{-1} l(x)=\{x\}$ if and only if both $f^{-1} f(x)=\{x\}$ and $g^{-1} g(x)=\{x\}$ so $S_{l}=S_{f} \cap S_{g}$.

Remark. The fact that $M(X)$ is a sublattice of $\mathrm{DEC}(X)$ and in particular the simple structure of $f \vee g$ as reflected in Definition 4.1 and Propositions 4.3 and 4.4 are the main advantages of H.I. spaces that are applied in the construction.

\section{A counterexample to the ChogoshVili CONJeCture}

Let $n \geq 2$ and $s \geq 2 n-1$ be integers and let $k$ be the greatest integer $\leq(s-1) /(n-1) \quad($ so $k \geq 2)$. Let $m=\left(\begin{array}{c}s \\ s-n+1\end{array}\right)(4(s-n)+3)$. Let $X$ be an $n$-dimensional H.I. space (which exists by [B]). We prove the following:

Theorem 5.1. There exists an embedding $g: X \rightarrow E_{m}$ and a decomposition $E_{m}=\sum_{a} \oplus E_{a}$, of $E_{m}$ into an orthogonal direct sum of $\left(\begin{array}{c}s \\ s-n+1\end{array}\right)$ linear subspaces $E_{a}$ of dimension $l=4(s-n)+3$ each, such that for every choice of $k$ of the subspaces $E_{a},\left\{E_{a_{i}}\right\}_{i=1}^{k}$ the composition $P g$ of $g$ with the canonical projection $P: E_{m} \rightarrow \sum_{i=1}^{k} \bigoplus E_{a_{i}}$ is 2-unstable.

Theorem 1.1 follows directly from Theorem 5.1 and the results of $\S 2$. For $k>2$ we obtain more information that in Theorem 1.1, namely large subspaces (sums of $k E_{a}$ 's) with large overlaps such that the projection of $g(X)$ on them is 2-unstable. Note however that by Theorem $2.2 *$ the projection of $g(X)$ onto one of the $(m-1)$-dimensional coordinate subspaces of $E_{m}$ must be $n$-stable. 
Proof of Theorem 5.1. As by a theorem of Hurewicz the light mappings form a dense $G_{\delta}$ set in $C\left(X, R^{n}\right)$ [Ku, p. 125] we can find in $C\left(X, R^{s}\right)$ an element $f^{\prime}=\left(f_{1}^{\prime}, f_{2}^{\prime}, \ldots, f_{s}^{\prime}\right)$ so that for every $1 \leq i_{1}<i_{2}<\cdots<i_{n} \leq s$ the element $f^{\prime}=\left(f_{i_{1}}^{\prime}, f_{i_{2}}^{\prime}, \ldots, f_{i_{n}}^{\prime}\right)$ of $C\left(X, R^{n}\right)$ is light. For each $1 \leq i \leq s$ let

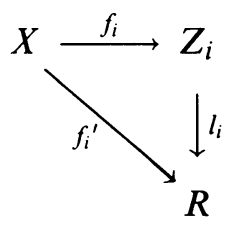

denote the monotone-light decomposition of $f_{i}^{\prime}$ (see [Ku, p. 184]). As $l_{i}$ is light it follows from the Hurewicz Theorem [H.W, p. 91] that $\operatorname{dim} Z_{i} \leq \operatorname{dim} R=1$; and as $f_{i}$ is monotone and has a one-dimensional range $f_{i}$ is a 2-unstable element of $M(X)$. Let $1 \leq i_{1}<i_{2}<\cdots<i_{n} \leq s$. As $f_{i} \leq f_{i}^{\prime}, \Lambda_{1 \leq j \leq n} f_{i_{j}} \leq$ $\Lambda_{1 \leq j \leq n} f_{i_{j}}^{\prime}$ and since the latter map is light so is the first. Hence $\Lambda_{1 \leq j \leq n} f_{i_{j}}$ is both monotone and light and thus an embedding. Let $S_{i}=S_{f_{i}}$ (see Definition 4.2). From Proposition 4.4 it follows that

5.1.

$$
X=S_{\bigwedge_{1 \leq j \leq n} f_{i_{j}}}=\bigcup_{j=1}^{n} S_{i_{j}}
$$

So we proved

5.2. The union of every $n$ of the $S_{i}$ 's is $X$. It follows that

5.3. Each point $x$ of $X$ belongs to $S_{i}$ for at least $s-n+1$ values of $i$. Indeed, let $x \in X$. If $x$ belongs only to $s-n S_{i}$ 's then the remaining $n S_{i}$ 's would not cover $X$ violating 5.2.

Let $A=\{a \subset\{1,2, \ldots, s\}$, cardinality of $a=|a|=s-n+1\} .5 .3$ is equivalent to

5.4. $X=\bigcup_{a \in A} \bigcap_{i \in a} S_{i}$.

We claim that

5.5. Every $k$ elements of $A$ have a nonempty intersection.

Proof.

$$
\begin{aligned}
\left|\{1,2, \ldots, s\} \backslash \bigcap_{i=1}^{k} a_{i}\right| & =\left|\bigcup_{i=1}^{k}\left(\{1,2, \ldots, s\} \backslash a_{i}\right)\right| \leq \sum_{i=1}^{k}\left|\{1,2, \ldots, s\} \backslash a_{i}\right| \\
& =k(n-1) \leq \frac{s-1}{n-1}(n-1)=s-1 .
\end{aligned}
$$

Hence $\bigcap_{i=1}^{k} a_{i}$ must contain at least one element.

For $a \in A$ let $\psi_{a} \in M(X)$ be defined by $\psi_{a}=\bigvee_{i \in a} f_{i}$ (see Definition 4.1). Let also $Y_{a}=\psi_{a}(X)$ and $\psi=\bigwedge_{a \in A} \psi_{a}: X \rightarrow \prod_{a \in A} Y_{a}=Y$.

5.6. Claim $\psi$ is an embedding.

Proof. By Proposition 4.4 and by 5.4, $S_{\psi}=\bigcup_{a \in A} S_{\psi_{a}}=\bigcup_{a \in A} \bigcap_{i \in a} S_{i}=X$.

5.7. Claim. Let $a_{1}, a_{2}, \ldots, a_{k}$ be $k$ elements of $A$. Then $\Lambda_{1 \leq j \leq k} \psi_{a_{j}}$ is 2-unstable. In particular, for $a, b$ in $A \psi_{a} \wedge \psi_{b}$ is 2-unstable. 
Proof. As $\psi_{a}=\bigvee_{i \in a} f_{i}$ and since the $f_{i}$ 's are 2-unstable $\left\{f_{i}\right\}_{i \in a} \subset \psi_{a}^{*}$ (see Definition 3.1).

By 5.5 there exists some $1 \leq i_{0} \leq s$ so that $i_{0} \in a_{j}$ for all $1 \leq j \leq k$. Then $f_{i_{0}} \in \psi_{a_{j}}^{*}$ for $1 \leq j \leq k$ and thus $f_{i_{0}} \in \bigcap_{1 \leq j \leq k} \psi_{a_{j}}^{*}$. By Proposition 3.1 $\Lambda_{1 \leq j \leq k} \psi_{a_{j}}$ is 2-unstable.

5.8. Claim. For each $a$ in $A \operatorname{dim} Y_{a} \leq 2(s-n)+1$.

Proof. Fix $a$ in $A$. For $i \in a$ let

$$
W_{i}=\left\{y \in Y_{a}: \psi_{a}^{-1}(y) \text { is a fiber of } f_{i}\right\} .
$$

Recall that $\psi_{a}=\bigvee_{i \in a} f_{i}$, so each fiber of $\psi_{a}$ is a fiber of one of the $f_{i}$ 's, $i \in a$. It follows that $Y_{a}=\bigcup_{i \in a} W_{i}$. Consider $\psi_{a}^{-1}\left(W_{i}\right) \subset X$. The mappings $\psi_{a}$ and $f_{i}$ induce the same decomposition on $\psi_{a}^{-1}\left(W_{i}\right)$ and hence $W_{i}=\psi_{a} \psi_{a}^{-1}(W)$ and $f_{i}\left(\psi_{a}^{-1}\left(W_{i}\right)\right.$ are homeomorphic. But $f_{i} \psi_{a}^{-1}\left(W_{i}\right) \subset Z_{i}$ and $\operatorname{dim} Z_{i}=1$. Hence $\operatorname{dim} W_{i} \leq 1$. Thus $Y_{a}$ is the union of $s-n+1$ 1-dimensional sets so $\operatorname{dim} Y_{a} \leq 2(s-n+1)-1=2(s-n)+1$.

Problem. Can one obtain a better estimate of $\operatorname{dim} Y_{a}$ ? In particular can one obtain 1-dimensional $Y_{a}$ 's?

Let us summarize what we obtained by now:

5.9. Given an $n$-dimensional H.I. continuum $X$ and $s \geq 2 n-1$, there exist $\left(\begin{array}{c}s \\ s-n+1\end{array}\right)$ monotone maps $\psi_{a}: X \rightarrow Y_{a}$, with $\operatorname{dim} Y_{a} \leq 2(s-n)+1$ so that $\psi=\bigwedge_{a} \psi_{a}: X \rightarrow \prod_{a} Y_{a}$ is an embedding and such that for every choice $a_{1}, a_{2}, \ldots, a_{k}$ of $k$ a's $\left(k=\right.$ greatest integer $\left.\leq \frac{s-1}{n-1}, k \geq 2\right) \bigwedge_{1 \leq j \leq k} \psi_{a_{j}}$ is 2-unstable.

Let now $h_{a}: Y_{a} \rightarrow E_{a}=R^{4(s-n)+3}$ be an embedding. Set $g_{a}=h_{a} \psi_{a}: X \rightarrow E_{a}$ and $g=\bigwedge_{a \in A} g_{a}: X \rightarrow \sum_{a \in A} \bigoplus E_{a}=E_{m}$ where $m=\left(\begin{array}{c}s \\ s-n+1\end{array}\right)(4(s-n)+3)$.

Note that since $h_{a}$ is an embedding $\psi_{a}$ and $g_{a}$ determine the same element of $M(X)$. It follows from 5.6 and 5.7 that $g$ is an embedding of $X$ in $E_{m}$, and that if $P$ is the canonical projection of $E_{m}$ onto the direct sum of any $k$ of the $E_{a}$ 's then $P g$ is 2-unstable. In particular if $L$ is a two-dimensional linear subspace of $E_{m}$ which is contained in $\sum_{1 \leq j \leq k} \oplus E_{a_{j}}=E$ then the canonical projection $Q$ of $E_{m}$ onto $L$ has no stable values on $g(X)$ since $Q$ factors through the projection $P$ of $E_{m}$ onto $E$ and thus $P \leq Q$ in $\operatorname{DEC}(g(X))$.

\section{REFERENCES}

[B] R. H. Bing, Higher dimensional hereditarily indecomposable continua, Trans. Amer. Math. Soc. 71 (1951), 267-273.

[B.P] C. Bessaga and A. Petczyński, Selected topics in infinite dimensional topology, PWN, Warsaw, 1975.

[Ch] G. Chogoshvili, On a theorem in the theory of dimensionality, Compositio Math 5 (1938), 292-298.

$\left[E_{1}\right] \quad$ R.. Engelking, Math. Rev. 90:k 54047.

$\left[\mathrm{E}_{2}\right] \quad$, Dimension theory, North-Holland, 1978.

[H.W] W. Hurewicz and H. Wallman, Dimension theory, Princeton Univ. Press, Princeton, N.J., 1941 .

[Ku] K. Kuratowski, Topology. II, Academic Press and PWN, 1968. 
[P] R. Pol, $A$ 2-dimensional compactum in the product of two 1-dimensional compacta which does not contain any rectangle, Ulam Quarterly (to appear).

[Si] K. Sitnikov, An example of two-dimensional set in three-dimensional Euclidean space allowing arbitrarily small deformations into a one-dimensional polyhedron and a certain new characterization of the dimension of sets in Euclidean spaces, Dokl. Akad. Nauk SSSR 88 (1953), 21-24.

Department of Mathematics, University of Haifa, Haifa 31999, IsRael

E-mail address: rsma711@haifauvm.bitnet. 